See discussions, stats, and author profiles for this publication at: https://www.researchgate.net/publication/3431165

\title{
Addressing Multiple Indicators on a Single Optical Fiber - Digital Signal Processing Approaches for Temperature Compensated Oxygen Sensing
}

Article in IEEE Sensors Journal · March 2004

DOI: 10.1109/JSEN.2003.822215 · Source: IEEE Xplore

CITATIONS

READS

11

2 authors, including:

3. Gerhard Alfred Holst

PCO AG

72 PUBLICATIONS 1,749 CITATIONS

SEE PROFILE

Some of the authors of this publication are also working on these related projects:

Project Frequency Domain Fluorescence Lifetime Imaging View project 


\title{
Addressing Multiple Indicators on a Single Optical Fiber-Digital Signal Processing Approaches for Temperature Compensated Oxygen Sensing
}

\author{
Christian Stehning and Gerhard A. Holst
}

\begin{abstract}
A digital signal processing approach for hybrid fiberoptical microoptodes based on the measurement of the luminescence lifetime of appropriate indicators is presented. Numerical algorithms are applied to resolve individual decay times in a heterogeneous luminescence emission to enable the application of optodes with multiple indicators combined on a single fiber tip.

We applied a hybrid optode that senses the oxygen concentration in line with the temperature, and used the latter information to compensate for the temperature drift of the oxygen measurement. We used a blue light-emitting diode to generate the excitation signal, and a standard photodiode with integrated pre-amplifier stage to detect the luminescence signal. Both signals were sampled using a stereo audio codec, and the further signal processing routines were entirely implemented on a commercially available digital signal processing evaluation board.
\end{abstract}

Index Terms-Digital signal processing (DSP), fiberoptical sensors, luminescence lifetime, oxygen optode, phase modulation technique, temperature compensation, temperature optode.

\section{INTRODUCTION}

$\mathbf{F}$ OR many sensor applications, especially in-situ, the simultaneous measurement of multiple parameters is favorable. Particularly, if parameters are interdependent, it is essential to measure all of them at the same time and position. An optical oxygen measurement based on the dynamic quenching of luminescence of appropriate indicators such as platinum-pentaphenylporphyrin (PFPP) is a very common example [9], [13], [14], [15], and [17]. Since the luminescence lifetime of the PFPP, like all luminescence indicators, is strongly influenced by the ambient temperature, it is necessary to determine the temperature at the sensor tip and compensate for the temperature drift of the oxygen measurement. We employed a hybrid optode that features a composition of two indicators (oxygen and temperature) with similar spectral characteristics on one single fiber tip, and resolved the individual decay times of the temperature and oxygen indicator from the heterogeneous sensor signal by employing a numerical algorithm discussed in

Manuscript received October 6, 2002; revised March 13, 2003. This work was supported by the Max Planck Society, Germany. The associate editor coordinating the review of this paper and approving it for publication was Prof. Ralph Etienne-Cummings.

C. Stehning was with the Max Planck Insitute for Marine Microbiology, Bremen, Germany. He is now with Philips Research, Hamburg, Germany (e-mail: christian.stehning@philips.com).

G. A. Holst was with the Max Planck Institute for Marine Microbiology, Bremen, Germany. He is now with PCO AG, Kelheim, Germany (e-mail: gerhard.holst@pco.de).

Digital Object Identifier 10.1109/JSEN.2003.822215 the following sections. In principle, various different indicators can be applied to the same fiber tip to measure multiple parameters, making the design of a "fiberoptical multimeter" possible. Besides the ease of use, the implementation on digital signal processors allows for a high degree of miniaturization suitable for in-situ applications.

\section{BASIC PRINCIPLES}

\section{A. Phase and Modulation Technique}

To detect the luminescence lifetime of a appropriate indicator, a phase and modulation technique is most practical for a digital measuring device. With the phase- and modulation technique, the luminophores are exited using a sinusoidally modulated light signal, $\mathrm{s}(\mathrm{t})$

$$
\mathrm{s}(\mathrm{t})=\mathrm{s}_{\mathrm{dc}}+\mathrm{s}_{\mathrm{ac}} \cdot \cos \left(\omega_{\bmod } \cdot \mathrm{t}\right)
$$

where $\omega_{\text {mod }}$ denotes the circular modulation frequency of the light intensity [4], $\mathrm{s}_{\mathrm{dc}}$ and $\mathrm{a}_{\mathrm{dc}}$ denote the dc component and modulation, respectively. The luminescence signal $[\mathrm{a}(\mathrm{t})]$ responds with the same circular frequency, but with a dc component $\mathrm{a}_{\mathrm{dc}}$, a (decreased) amplitude $\mathrm{a}_{\mathrm{ac}}$, and a phase shift $\varphi$ [4]

$$
\mathrm{a}(\mathrm{t})=\mathrm{a}_{\mathrm{dc}}+\mathrm{a}_{\mathrm{ac}} \cdot \cos \left(\omega_{\bmod } \cdot \mathrm{t}-\varphi\right)
$$

where

$$
\mathrm{a}_{\mathrm{ac}}=\frac{\mathrm{s}_{\mathrm{ac}}}{\sqrt{1+(\omega \cdot \tau)^{2}}}
$$

and

$$
\varphi=\tan ^{-1}(\omega \cdot \tau)
$$

If the luminescence emission is caused or at least dominated by a single homogeneous emission, the decay constant $(\tau)$ can either be calculated from the measured amplitude $\mathrm{a}_{\mathrm{ac}}$ (3), or from the phase shift $\varphi$ (4). For fiber sensor applications, it is applicative to measure the phase shift, as the measurable light intensity is disturbed by various factors, i.e., the fiber bend.

After conversion from light intensity to an electrical signal by appropriate photodetectors (photomultiplier tube or photodiode), electrical highpass filters are applied to eliminate the dc component and low temporal frequency signals introduced by background light (sun or artificial light) coupled into the fiber tip. In the measuring setup presented in Section IV, a single-stage passive filter (RC-network, cutoff frequency $\mathrm{f}_{\mathrm{C}}=500 \mathrm{~Hz}$ ) was applied remove the dc component $\mathrm{a}_{\mathrm{dc}}$ and low frequency components $(120 \mathrm{~Hz}$ plus harmonics introduced by fluorescent lamps). 


\section{B. Measuring Phase Shifts in the Frequency Domain}

With a digital signal processing system, the phase shift $\varphi$ is preferably determined in the frequency domain (FD). The sinusoidal excitation- and response signals are sampled and transformed into the FD using a fast Fourier transform (FFT) algorithm. The quotient of the transformed response- and excitation signal yields the complex transfer function of the indicator at the modulation frequency [5]

$$
\mathrm{H}(\mathrm{k})=\frac{\operatorname{FFT}\left\{\mathrm{a}\left(\mathrm{k} \cdot \mathrm{f}_{\text {sample }}\right)\right\}}{\operatorname{FFT}\left\{\mathrm{s}\left(\mathrm{k} \cdot \mathrm{f}_{\text {sample }}\right)\right\}}
$$

where $\mathrm{k}$ denotes the index of the samples taken and $\mathrm{f}_{\text {sample }} \mathrm{de-}$ notes the sampling frequency. As only discrete frequencies appear in the $\mathrm{H}(\mathrm{k})$ data array, a modulation frequency that lies on the frequency grid must be used.

The phase shift and the measured luminescence decay time is then calculated from the real- and imaginary part of the complex transfer function (6)

$$
\tan (\varphi)=\omega_{\bmod } \cdot \tau=\frac{\operatorname{im}\{\mathrm{H}(\mathrm{k})\}}{\operatorname{re}\{\mathrm{H}(\mathrm{k})\}} .
$$

As only a fraction that matches the modulation frequency has an impact on the measurement, the sensor noise is inherently bandpass-filtered [6].

\section{Composition of a Hybrid Sensor Signal}

If multiple fluorescence indicators are combined within the sensor material at the fiber tip, the resulting sensor signal is a composition of the signal of each individual indicator. Due to the spectral Stokes shift between the excitation- and luminescence light, we will presume that both indicators do not interact, in particular the luminescence signal of one indicator does not stimulate a luminescence emission of the other. For a hybrid sensor, such as the oxygen/temperature optode that will be further discussed in Section IV, we further presume that both indicators have similar spectral characteristics, and thus pass the common optical path uniformly. For the resulting light intensity at the photodiode detector, we then have a linear combination of the involved indicator response signals. When using a sinusoidally modulated excitation signal, this again yields a sinusoidal response signal with the same frequency as the excitation signal [1]

$$
\begin{aligned}
\mathrm{a}_{\mathrm{m}}(\mathrm{t}) & =\sum_{\mathrm{n}=1}^{\mathrm{N}} \mathrm{a}_{\mathrm{n}}(\mathrm{t}) \\
& =\mathrm{a}_{\mathrm{m}, \mathrm{dc}}+\mathrm{a}_{\mathrm{m}, \mathrm{ac}} \cdot \cos \left(\omega_{\bmod } \cdot \mathrm{t}-\Theta_{\mathrm{m}}\right)
\end{aligned}
$$

$\mathrm{a}_{\mathrm{m}, \mathrm{ac}}=\sqrt{\left(\sum_{\mathrm{n}=1}^{\mathrm{N}} \mathrm{a}_{\mathrm{ac}, \mathrm{n}} \cdot \sin \left(\varphi_{\mathrm{n}}\right)\right)^{2}+\left(\sum_{\mathrm{n}=1}^{\mathrm{N}} \mathrm{a}_{\mathrm{ac}, \mathrm{n}} \cdot \cos \left(\varphi_{\mathrm{n}}\right)\right)^{2}}$

and

$$
\tan \left(\Theta_{\mathrm{m}}\right)=\frac{\sum_{\mathrm{n}=1}^{\mathrm{N}} \mathrm{a}_{\mathrm{ac}, \mathrm{n}} \cdot \sin \left(\varphi_{\mathrm{n}}\right)}{\sum_{\mathrm{n}=1}^{\mathrm{N}} \mathrm{a}_{\mathrm{ac}, \mathrm{n}} \cdot \cos \left(\varphi_{\mathrm{n}}\right)}
$$

If two indicators are combined, the calculation of a lifetime from the measured phase angle $\left(\Theta_{\mathrm{m}}\right)$ or amplitude $\left(\mathrm{a}_{\mathrm{m}, \mathrm{ac}}\right)$ using (4) or (3) results in a measured or "apparent" lifetime $\tau_{\mathrm{m}}$, but the luminescence emissions that are actually present in the sensor signal cannot be resolved from a measurement at a single modulation frequency.

Alternative approaches separate the luminescence emission in the spectral domain using optical filters [17], but due to the spectral overlap of the absorption and emission of many indicators, this is a difficult task. Therefore, we decided to separate multiple individual lifetimes in a heterogeneous luminescence emission not in the spectral domain, but in the temporal domain by applying numerical algorithms presented in the following section.

\section{NumericAl APPROACHES TO RESOlVE MultiPle DeCAY \\ TIMES IN A HETEROGENEOUS LUMINESCENCE EMISSION}

In the case of two extremely widely spaced decay times, the luminescence lifetimes are calculated by a plot of the luminescence light intensity decay $(\log (\mathrm{I}(\mathrm{t}))$ following an excitation versus time [3]. The first luminescence component (short $\tau$ ) is determined by sampling the heterogeneous luminescence decay shortly after the excitation (assuming the long decay component to be constant within the sampling interval), whereas the second luminescence component (long $\tau$ ) is determined by sampling the luminescence decay after a time delay that ensures that the luminescence from the first component has entirely died away. However, this simple but effective approach is not suitable for rather closely spaced decay constants as displayed by our hybrid optode discussed in Section IV.

Here, the presence of two or more individual lifetimes in a heterogeneous luminescence emission is detected in the frequency domain by applying a phase- and modulation technique at a set of different modulation frequencies [1], [2], [4], and [10].

\section{A. Two-Frequency Moment Method}

A closed-form procedure for the determination of the decay constants and the relative contributing intensities of the $N$ independent components of a heterogeneous fluorescence emission at $N$ excitation frequencies is called the moment method and has been described by Gregorio Weber in 1980 [1]. To determine two individual lifetimes, the measurement of the complex transfer function $\mathrm{H}(\mathrm{j} \omega)$ at two different modulation frequencies is necessary. Assuming that the decay times remain constant, the measurements can be done successively, but an advanced approach is to apply both modulation frequencies simultaneously [6].

In the example shown in Fig. 1, two excitation frequencies at $\mathrm{f}_{\text {mod, } 1}=3 \mathrm{kHz}$ and $\mathrm{f}_{\bmod , 2}=21 \mathrm{kHz}$ were used, using the largest possible bandwidth for the applied audio codec. The decay times were $\tau_{1}=3 \mu \mathrm{s}$ and $\tau_{2}=20 \mu \mathrm{s}$, at a sampling rate of $\mathrm{f}_{\text {sample }}=48 \mathrm{kHz}$.

To resolve two decay times, the first four moments $\left(m_{0}-m_{3}\right)$ of the complex transfer function (5) are calculated. The zero and even moments arise from a linear combination of the real part of the transfer function, whereas the odd moments arise 


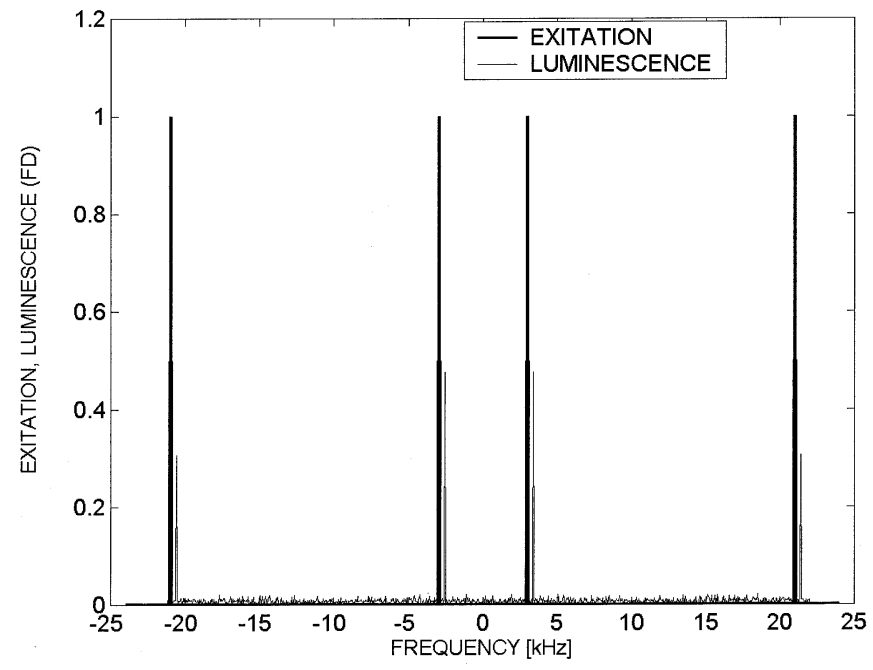

Fig. 1. Two-frequency moment method: excitation and luminescence signal in the frequency domain.

from a linear combination of the imaginary parts at the given modulation frequencies [1]

$$
\begin{aligned}
\mathrm{m}_{\mathrm{k}=0,2}= & \pm \mathrm{p}_{1}^{(2-\mathrm{k})} \cdot \operatorname{re}\left\{\mathrm{H}\left(\mathrm{i}_{\bmod 1}\right)\right\} \\
& \mp \mathrm{p}_{2}^{(2-\mathrm{k})} \cdot \mathrm{re}\left\{\mathrm{H}\left(\mathrm{i}_{\bmod 2}\right)\right\} \\
\mathrm{m}_{\mathrm{k}=1,3}= & \pm \mathrm{p}_{1}^{(2-\mathrm{k})} \cdot \operatorname{im}\left\{\mathrm{H}\left(\mathrm{i}_{\bmod 1}\right)\right\} \\
& \mp \mathrm{p}_{2}^{(2-\mathrm{k})} \cdot \operatorname{im}\left\{\mathrm{H}\left(\mathrm{i}_{\bmod 2}\right)\right\}
\end{aligned}
$$

where

$$
\mathrm{p}_{\mathrm{i}}=\frac{\omega_{\mathrm{i}}}{\omega_{\mathrm{ref}}}
$$

with $\omega_{\text {ref }}$ being a reference frequency that may be freely selected. The symmetric functions $\left(\Theta_{1}, \Theta_{2}\right)$ of the lifetimes become

$$
\Theta_{1}=\frac{m_{3} m_{0}-m_{2} m_{1}}{m_{2} m_{0}-m_{1}^{2}}, \quad \Theta_{2}=\frac{m_{3} m_{1}-m_{2}^{2}}{m_{2} m_{0}-m_{1}^{2}} .
$$

Finally, both decay times $\left(\tau_{1}, \tau_{2}\right)$ are calculated according to (13)

$$
\tau_{1,2}=\frac{\frac{\Theta_{1}}{2} \pm \sqrt{\frac{\Theta_{1}^{2}}{4}-\Theta_{2}}}{\omega_{\text {ref }}} .
$$

A detailed deduction of the moment method is given elsewhere [1], but it is important to consider the simplicity of its application. The numerical calculations are simple enough to be performed in line with the data acquisition. At a sample rate of $\mathrm{f}_{\text {sample }}=48 \mathrm{kHz}$, the acquisition of 1024 samples for the FFT takes approximately $22 \mathrm{~ms}$, whereas the FFT and further multiplications according to (10)-(13) are performed within a few hundred microseconds.

\section{B. Multifrequency Fit}

Unlike the moment method, the multifrequency fit [4] is not a closed-form calculation. The mathematical model for the phase angle raised by a binary indicator combination is fittet to the
FD data measured at a set of different modulation frequencies (Fig. 2).

The useful modulation frequencies are those for which the phase angle is sufficiently frequency-dependent (4) and there is still measurable modulation (3). For instance, the model for the phase frequency response of a two component indicator combination is then determined by transforming the individual impulse responses into the frequency domain and rearranging the resulting function toward the phase angle [2], [6]

$$
\tan (\Theta)=\frac{\left(\frac{\mathrm{f}_{1} \cdot \omega \cdot \tau_{1}}{\left(\omega \cdot \tau_{1}\right)^{2}+1}\right)+\left(\frac{\left(1-f_{1}\right) \cdot \omega \cdot \tau_{2}}{\left(\omega \cdot \tau_{2}\right)^{2}+1}\right)}{\left(\frac{f_{1}}{\left(\omega \cdot \tau_{1}\right)^{2}+1}\right)+\left(\frac{\left(1-f_{1}\right)}{\left(\omega \cdot \tau_{2}\right)^{2}+1}\right)} .
$$

The goodness-of-fit between the model and the measured data is judged by deviations between the measured phase angles $\varphi_{\mathrm{i}}$ and the calculated curve $\Theta\left(\omega ; \tau_{1}, \tau_{2}, f_{1}\right)$ [7]

$$
\aleph^{2} \equiv \frac{1}{\nu} \sum_{\mathrm{i}=1}^{\mathrm{N}}\left(\frac{\varphi_{\mathrm{i}}-\Theta\left(\omega_{\mathrm{i}} ; \tau_{1}, \tau_{2}, \mathrm{f}_{1}\right)}{\sigma \Theta_{\mathrm{i}}}\right)^{2} \rightarrow \min
$$

where $\nu$ is the number of degrees of given by the number of measurements (twice the number of frequencies $N$, since the real and imaginary parts are calculated), minus the number of variable parameters $\left(\tau_{1}, \tau_{2}, \mathrm{f}_{1}\right)$. The standard deviation of the measured phase angle, $\sigma \Theta_{\mathrm{i}}$, can be obtained by repetively measuring the phase angle at the same modulation frequency. The model function is then adjusted by altering the fit parameters $\tau_{1}, \tau_{2}$, and $f_{1}$. Once the error function $\chi^{2}$ has reached its minimum, the recovered parameter values $\tau_{1}$ and $\tau_{2}$ are evaluated [6]. Because of the nonlinear relationship between the fit parameters $\tau_{1}, \tau_{2}$, and $\mathrm{f}_{1}$ and the resulting model function, the minimization of the $\chi^{2}$ function must proceed iteratively. A well established method for such a nonlinear least-square approximation is the Levenberg-Marquard iteration [7]. The processing time for the multifrequency fit is $22 \mathrm{~ms}$ for data collection (1024 samples at $\mathrm{f}_{\text {sample }}=48 \mathrm{kHz}$ ) plus $200-250 \mathrm{~ms}$ processing time for iteratively fitting the two-exponential model function (14) to the collected data (Matlab 4.1 on a Pentium III processor at $600 \mathrm{MHz}$ ).

The advance of a least-squares-approach over the moment method is that the precision of the resolved fit parameters is less sensitive to the uncertainty of the measured values, which has been discussed in detail in [4]. For this reason, we implemented the multifrequency fit into our measuring system which is further discussed in Section IV.

\section{On the Performance of the Multifrequency Fit to Resolve Multiple Lifetimes in a Heterogeneous Fluorescence Emission}

Prior to implementation, an estimate of the achievable precision of the multifrequency is important. Unfortunately, a closed-form calculation of the expected uncertainties of the resolved parameters $\tau_{1}, \tau_{2}$, and $\mathrm{f}_{1}$ from the uncertainty $\sigma \theta$ of the measured values (phase angles) is difficult. This is because the uncertainty of the fit parameters do not directly correlate with the uncertainty of the measured values when using a least-squares approach [4]. The resolved parameter values can be expected sensitive to $\sigma \Theta$ only if the number of data are just adequate to determine the parameter values, but not for a large 


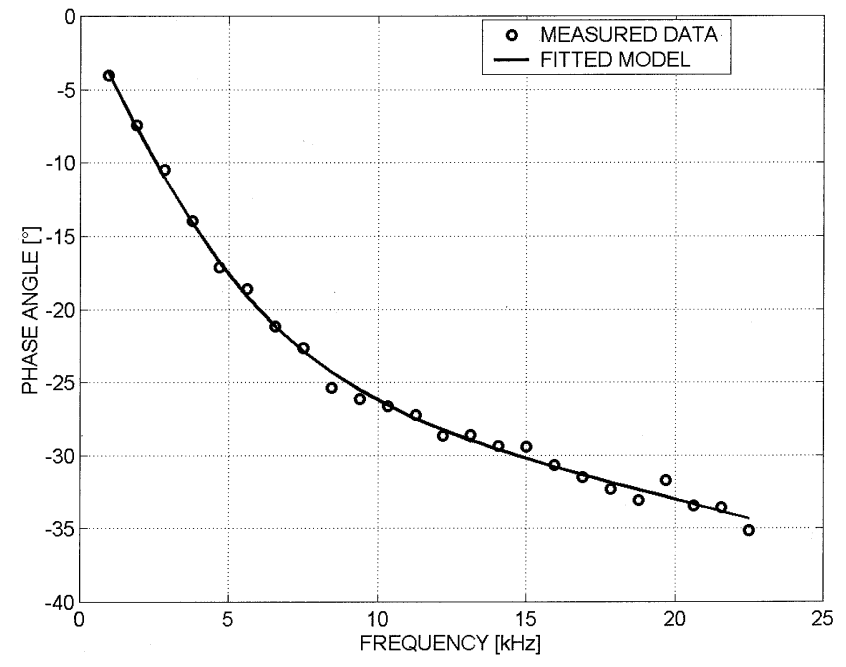

Fig. 2. Multifrequency fit: Measured phase angles and fitted model function in the frequency domain.

number of measurements (at different modulation frequencies) commonly used for the multifrequency fit. Further, we cannot assume $\sigma \theta$ to be constant for all measurements, as it strongly alters the signal-to-noise ratio (SNR) achieved at different modulation frequencies. Therefore, an approach presented in [4] is not to estimate the uncertainty of resolved parameters, but to judge the general correctness of the model function on the value of $\chi^{2}$ in (15) itself. For an appropriate model and random noise, $\chi^{2}$ is expected to be near unity, otherwise one should consider whether $\chi^{2}$ is adequate to reject the model. Rejection is judged from the probability that random noise could be the origin of the value of $x^{2}$ [4]. However, we achieved a prediction of the achievable precision of the multifrequency fit at different parameter values ( $\tau_{1}$ and $\tau_{2}$ ) using a numerical simulation. The corresponding sensor signal of a biexponential decay combination ranging from $1 \mu \mathrm{s}$ to $6 \mu \mathrm{s}\left(\tau_{1}\right)$ and from $10 \mu \mathrm{s}$ to 100 $\mu \mathrm{s}\left(\tau_{2}\right)$ were simulated, matching the individual decay times displayed by the hybrid optode presented in Section IV. For simplicity, the noise present in the sensor signal was assumed to be an additive white gaussian noise process, at an SNR of

$$
\mathrm{SNR}=20 \cdot \log \left(\frac{\text { signal amplitude }}{\text { noise amplitude }}\right)=9 \mathrm{~dB} .
$$

This assumption was justified by first measuring the signal amplitude of the hybrid optode presented in Section IV, and then measuring the amplitude of the noise only with the excitation light switched off (cf. Fig. 3).

After adding the corresponding random noise signal to the simulated sensor signal, the individual lifetimes were recalculated from the resulting signal using the multifrequency fit. The simulation was repeated ten times at six different values for $\tau_{1}$ ( temperature indicator) and one hundred different values for $\tau_{2}$ ( oxygen indicator). The standard deviations $\sigma \tau_{2}$ (in $\mu \mathrm{s}$ ) of the resolved values for $\tau_{2}$ are plotted in Fig. 4. For improved visualization, the determined values for $\sigma \tau_{2}$ were cut at a maximum value of $20 \mu \mathrm{s}$.

As indicated by the simulation result, the resolution of multiexponential decay times becomes more difficult as the decay

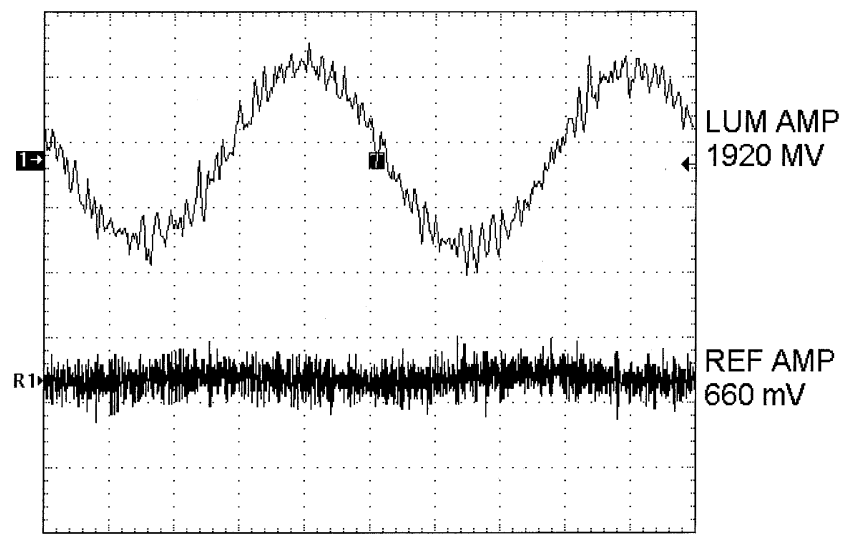

Fig. 3. Measured sensor signal of a hybrid optode.

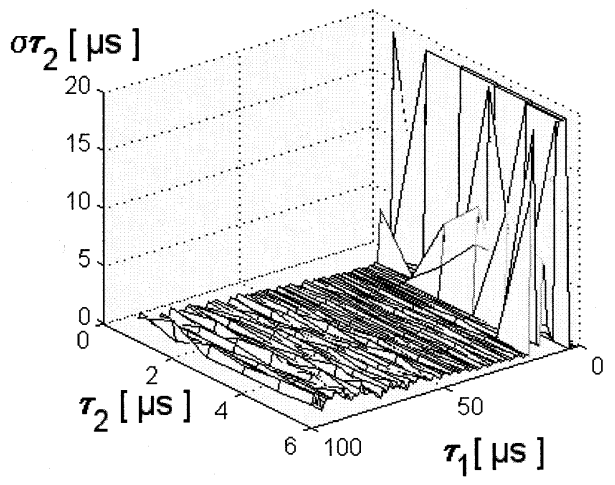

Fig. 4. Multifrequency fit: standard deviation $\sigma \tau 2$ of the resolved decay time $\tau_{2}$ ( oxygen indicator) for a two-component model function.

times become more closely spaced. Under given noise conditions, the multifrequency fit only yields reliable results $\left(\sigma \tau_{2}<\right.$ $0.5 \mu \mathrm{s})$ for $\tau_{2} \gg \tau_{1}$, namely $20 \mu \mathrm{s}$. This result is in good compliance with [4], where it was previously stated that it is difficult to resolve decay times that are less than twofold different.

Another problem is whether the a model function assuming two luminescence components (14) is a sufficient approximation of the realistic sensor characteristic of a hybrid optode employing two indicators. Most indicators display, for example, more than one decay time themselves, due to their inhomogeneous composition or interaction of the luminophores within the immobilization matrix material. For the available version of a hybrid oxygen/temperature optode discussed in Section IV, we achieved an optimal correlation coefficient between the measured data and the model function when assuming three luminescence components, with the decay time of the third component held constant at $\tau_{3}=20 \mu \mathrm{s}$. On the other hand, it is most difficult to resolve three luminescence components out of the measured data at the given noise condition, due the greater number of fit parameters $\left(f_{1}, \tau_{1}, \tau_{2}, f_{3}, \tau_{3}\right)$ that determine the model function [6].

As indicated by the simulation results in Fig. 5, the multifrequency fit does not yield reliable results for a three-component exponential model function under given noise conditions. For practical applications, it is therefore more desirable to improve the homogeneity of the indicator composition than increasing the complexity of the model function. 


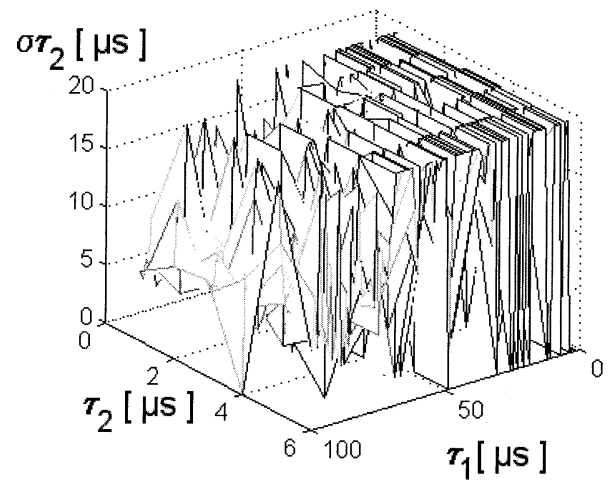

Fig. 5. Multifrequency fit: standard deviation $\sigma \tau 2$ of the resolved decay time $\tau_{2}$ ( oxygen indicator) for a three-component model function.

\section{APPLICATION-THE TEMPERATURE COMPENSATED FIBEROPTICAL OXYGEN SENSOR}

\section{A. Principle of Temperature Compensation}

A detailed description of how to compensate for the temperature effect of a fiberoptical oxygen measurement is given in [5]. In short, a temperature dependency of the parameters $\tau_{0}$, which is the decay time in the absence of oxygen, and $\mathrm{K}_{\mathrm{SV}}$, which is the quenching coefficient of the luminescence indicator, has to be taken into account in the experimentally modified Stern-Volmer equation (18) that describes the characteristic curve of an oxygen microoptode [9]

$$
\tau=\tau_{0}(\mathrm{~T}) \cdot\left(\frac{\mathrm{f}_{1}(\mathrm{~T})}{1+\mathrm{K}_{\mathrm{SV}}(\mathrm{T}) \cdot\left[\% \mathrm{O}_{2}\right]}+\left\{1-\mathrm{f}_{1}(\mathrm{~T})\right\}\right) .
$$

To calibrate for the characteristic curve of the oxygen indicator, the temperature at the fiber tip has to be measured prior to the calculation of the oxygen concentration. For this reason, a hybrid microoptode has been applied that is capable to sense the temperature in line with the oxygen concentration.

\section{B. Hybrid Microoptode}

The hybrid microoptode developed for this application is based on a common multimode graded index fiber $(100 / 140 \mu \mathrm{m})$ supplied with a ST-plug at the measuring system side. The sensing layer consists of two different types of sensing particles that were immersed in a polymer matrix.

Due to its comparable long decay time (24 $\mu$ s at $20 \%$ vol $\mathrm{O}_{2}$ and $20{ }^{\circ} \mathrm{C}$ ambient temperature) and high signal intensity, platinum-pentaphenylporphyrin (PFPP) immobilized in polysterene ([13], [15], and [17]) with an average particle diameter of $8 \mu \mathrm{m}$ [Fig. 6(a)] was applied as an oxygen indicator. Ruthenium(II)-tris-(1, 10-phenanthroline) (Ru(phen)) (Aldrich, 34, 371-4) was used as a temperature indicator due to the temperature dependency of its luminescence lifetime [11]. To reduce its cross-sensitivity to oxygen, the Ru(phen) was embedded in a poly(acrylonitrile) (PAN-) matrix ([8], [9], [11]-[13], [16], and [17]) to prevent the access of oxygen. The decay time of $\mathrm{Ru}\left(\right.$ phen) varies between $4.4 \mu \mathrm{s}\left(5^{\circ} \mathrm{C}\right)$ and $3.2 \mu \mathrm{s}\left(55^{\circ} \mathrm{C}\right)$. The coated sensor material was ground to particles with a mean diameter of $40 \mathrm{~nm}$ [Fig. 6(b)]. The dispersion of both sensing particles in an adapted polystyrene solution was used to dip-coat the tapered fiber tip.

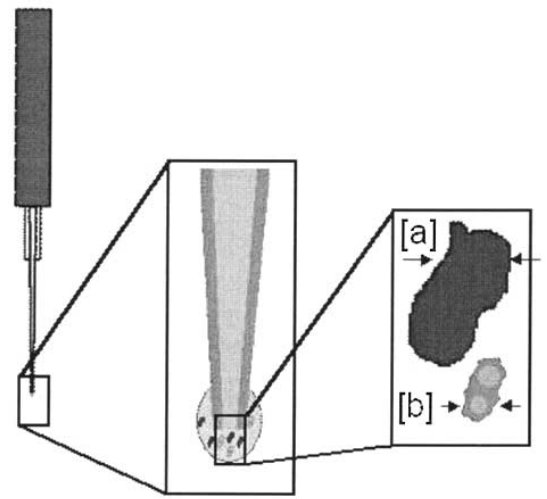

Fig. 6. Preparation of a hybrid sensor.

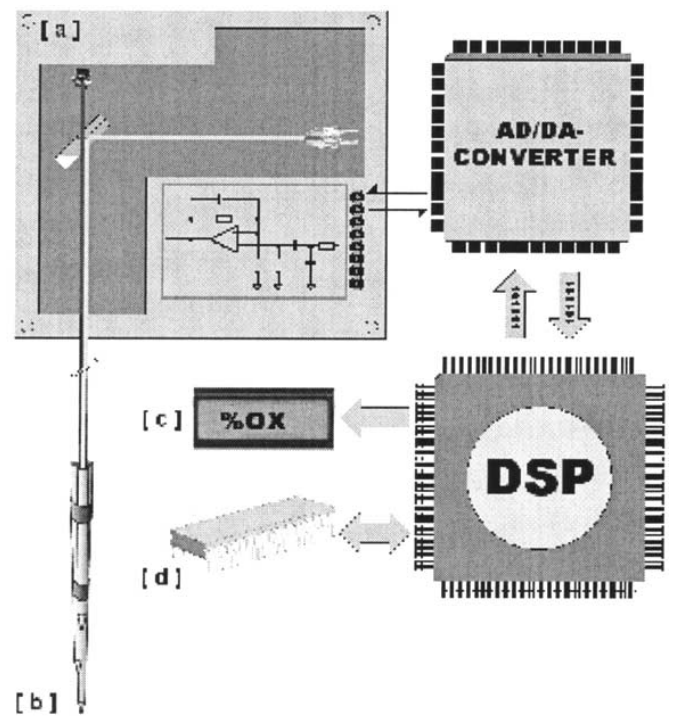

Fig. 7. Functional units of the measuring system.

As both indicators display similar spectral characteristics (absorption at $\lambda \approx 500 \mathrm{~nm}$, emission at $\lambda \approx 580 \mathrm{~nm}$ wavelength), their luminescence signals pass the optical path as well as the optoelectronical converter uniformly and yield a linear combination as assumed in (7).

\section{Measuring System}

A schematic overview of the measuring system that was used for the hybrid sensor is shown in Fig. 7.

An AD/DA converter (16 Bit Stereo Audio Codec AD1847, Analog Devices, USA) controls a voltage-controlled current source that drives the blue LED (Nichia Co., 505-nm wavelength). Within the optoelectronic module shown in Fig. 7(a), a wavelength selective mirror (R61 by Balzer/Linos Photonics +2 mm OG590 from ITOS) that reflects wavelengths below $590 \mathrm{~nm}$ and passes higher spectra is used to direct the modulated excitation light into the fiber with the luminescence indicator applied on the fiber tip [Fig. 7(b)]. The Stokes-shifted luminescence emission light signal passes the mirror into a detector diode with an integrated preamplifier. After amplification (OPA2604, Burr-Brown, gain factor 50) a passive, double-stage bandpass filter (lower cutoff frequency $500 \mathrm{~Hz}$, upper cutoff frequency $100 \mathrm{kHz}$ ) is applied. Two programmable 
gain amplifiers (AD712, Analog Devices with programmable potentiometer, Xircom X9250) are used for additional amplification. The voltage signal used to modulate the excitation light passes an identical analog signal processing stage and serves as a reference signal. Finally, both channels are sampled by the stereo audio codec and fed into the 32-bit floating point DSP (ADSP 21061, Analog Devices, USA) that performs further signal processing. The calculated oxygen concentration is then displayed [Fig. 7(c)] and stored in a nonvolatile data memory [Fig. 7(d)].

\section{Sensor Calibration Procedure}

The hybrid optode is calibrated using a double-walled glass mixing vessel to adjust the temperature and oxygen concentration. The outer cycle is filled with water of a fixed temperature, while the inner circle contains salt water that is flushed by a gas mixture through a batch. A gas mixing pump is used to set an oxygen/nitrogen compound with an adjustable oxygen concentration. Ten different oxygen concentrations ranging from 0 [\%vol] to 100 [\%vol] were set as shown in Table I. The measurement was repeated at six different temperatures ranging from $5{ }^{\circ} \mathrm{C}$ to $55^{\circ} \mathrm{C}$.

The luminescence lifetimes of the temperature indicator $\left(\tau_{1}\right)$ and the oxygen indicator $\left(\tau_{2}\right)$ were resolved using the multifrequency fit at 19 modulation frequencies ranging from $3 \mathrm{kHz}$ to $21 \mathrm{kHz}$. The resolved luminescence lifetime $\tau_{1}$ of the temperature indicator is then correlated with the set temperature using a linear fit. Then the temperature dependent parameters $\tau_{0}(\mathrm{~T}), \mathrm{f}_{1}(\mathrm{~T})$, and $\mathrm{K}_{\mathrm{SV}}(\mathrm{T})$ in (18) were determined by fitting the Stern-Volmer-equation to the resolved luminescence lifetimes $\tau_{2}$ of the oxygen indicator at different temperatures.

After completing the calibration process, $\tau_{0}(\mathrm{~T}), \mathrm{f}_{1}(\mathrm{~T})$, and $\mathrm{K}_{\mathrm{SV}}(\mathrm{T})$ were passed to the DSP operation software. A temperature compensated oxygen measurement was then performed by rearranging (18) toward the oxygen concentration [\%O2] at six different temperatures ranging from $5{ }^{\circ} \mathrm{C}$ to $55^{\circ} \mathrm{C}$. Despite the PAN-isolation of the temperature sensor (Ruphen) described in Section IV, the observed luminescence lifetime of the temperature indicator of the preliminary sensor version was not completely independent of the oxygen. For this reason, the actual parameter values were determined using an iterative procedure. First, an approximation to the temperature at the fiber tip was determined by measuring $\tau_{1}$ and correlating it to the temperature using the characteristic curve, initially assuming an oxygen concentration value of $20 \%$ [vol]. Then, the oxygen concentration estimate was improved by evaluating $\tau_{2}$ and correlating it to the Stern-Volmer equation (18). This procedure was repeated until the difference between the successive estimates was below an acceptable threshold.

\section{RESULTS}

The results of the temperature compensated oxygen measurement is shown in Table I and Fig. 8, respectively.

The column O2_SET denotes the oxygen concentration (\% vol) set with the gas mixing pump, O2_M_MIN, O2_M_MAX, and $\mathrm{O} 2$ M_MEAN denote the minimum, maximum, and mean value of the measured oxygen concentration measured

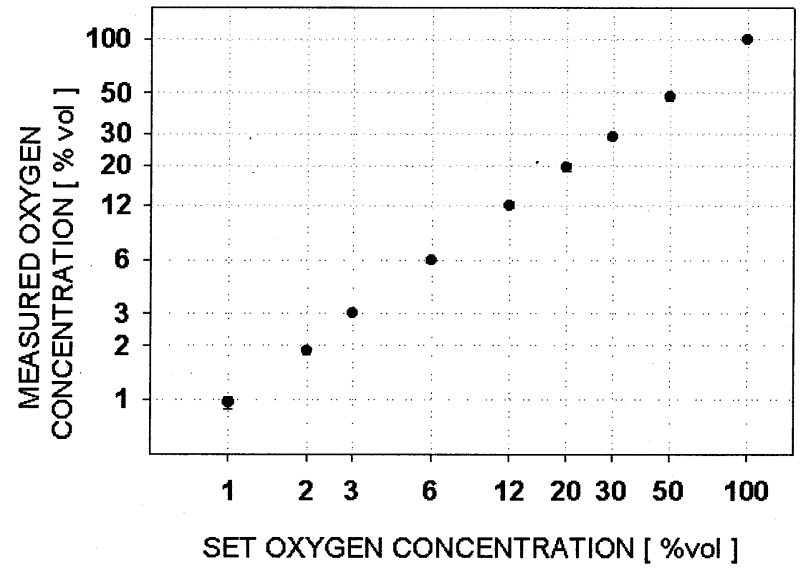

Fig. 8. Result of the oxygen measurement with the hybrid optode (temperature compensated).

TABLE I

Results OF THE OXYgen MEASUREMENT With THE Hybrid OPTODE (TEMPERATURE COMPENSATED)

\begin{tabular}{c|c|c|c|c|c}
\hline O2_SET & O2_M_MIN & O2_M_MAX & O2_M_MEAN & STD DEV & TAU2 \\
\hline & & & & & \\
\hline 0 & 0,00 & 0,50 & 0,15 & 0,16 & 52,6 \\
\hline 1 & 0,90 & 1,10 & 0,97 & 0,07 & 46,3 \\
\hline 2 & 1,80 & 2,00 & 1,88 & 0,09 & 41,8 \\
\hline 3 & 2,90 & 3,20 & 3,02 & 0,11 & 38,7 \\
\hline 6 & 5,90 & 6,40 & 5,98 & 0,19 & 32,3 \\
\hline 12 & 11,40 & 12,80 & 12,00 & 0,49 & 25,5 \\
\hline 20 & 18,40 & 21,20 & 19,55 & 1,00 & 21,9 \\
\hline 30 & 26,70 & 30,10 & 28,95 & 1,14 & 20,4 \\
\hline 50 & 44,50 & 50,90 & 47,87 & 2,45 & 18,1 \\
\hline 100 & 97,60 & 99,90 & 99,52 & 0,86 & 14,5 \\
\hline \multicolumn{7}{|c|}{}
\end{tabular}

with the temperature compensated optode setup. TAU2 is the corresponding decay time of the oxyen indicator (at $15^{\circ} \mathrm{C}$ temperature). The standard deviation (STD_DEV) is equal or less than $1 \%$ [vol] below a threshold of $20 \%$ vol $(\sim 100 \%$ air saturation), which is a sufficient range for most applications. For oxygen concentrations higher than $20 \%$ vol $\left(\tau_{2}<22 \mu\right.$ s), the difficulty of resolving closely spaced luminescence lifetimes previously discussed in Section III shows as $\tau_{2}$ approaches $\tau_{1}$ ( $\sim 4 \mu \mathrm{s}$ ). For comparison, the result of an oxygen measurement without temperature compensation, but with $\tau_{0}(\mathrm{~T}), \mathrm{f}_{1}(\mathrm{~T})$, and $\mathrm{K}_{\mathrm{SV}}(\mathrm{T})$ assumed fixed at their values at $20{ }^{\circ} \mathrm{C}$ is shown in Table II and Fig. 9, respectively.

Beside a standard deviation of more than $6 \%$ vol, a systematic error occurs (calculated oxygen concentration too high) if the altering temperature is not taken into account.

\section{CONCLUSION}

The first results of the oxygen-temperature hybrid microoptode prove the achieved performance of the proposed numerical solutions for multiple luminescence lifetime measurements. The realization of temperature compensated oxygen microoptode measurements, that greatly enhance in-situ applications where the fiber tip temperature can hardly be controlled, demonstrate the quality of the presented approach. 


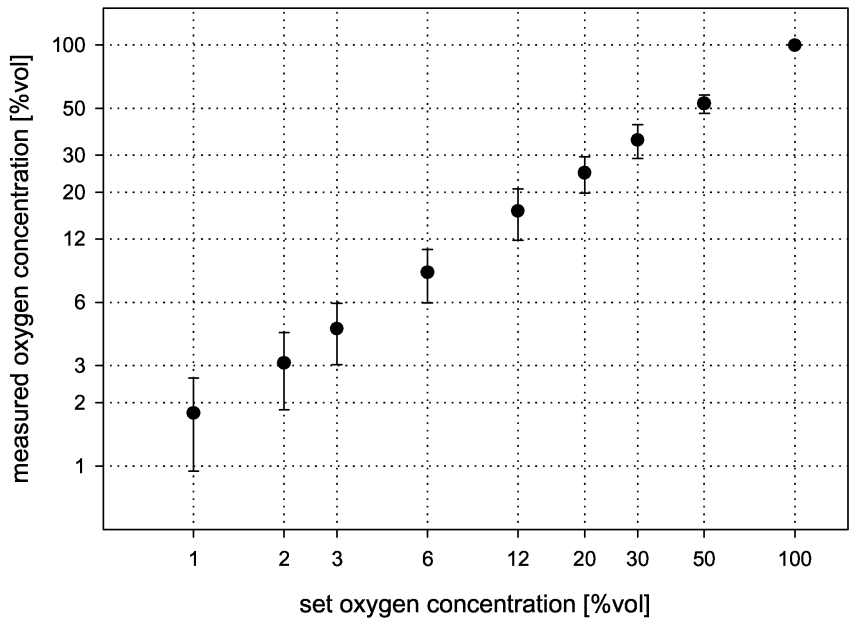

Fig. 9. Results of the oxygen measurement with the hybrid optode (without temperature compensation).

TABLE II

RESULTS OF THE OXYGEN MEASUREMENT WITH THE HybRID OPTODE (WITHOUT TEMPERATURE COMPENSATION)

\begin{tabular}{c|c|c|c|c|c}
\hline O2_SET & O2_M_MIN & O2_M_MAX & O2_M_MEAN & STD DEV & TAU2 \\
\hline & & & & & \\
\hline 0 & 0,00 & 2,20 & 0,72 & 0,72 & 52,6 \\
\hline 1 & 0,60 & 3,10 & 1,78 & 0,84 & 46,3 \\
\hline 2 & 1,40 & 4,90 & 3,08 & 1,23 & 41,8 \\
\hline 3 & 2,40 & 6,40 & 4,48 & 1,45 & 38,7 \\
\hline 6 & 4,90 & 11,80 & 8,32 & 2,36 & 32,3 \\
\hline 12 & 9,90 & 23,40 & 16,27 & 4,43 & 25,5 \\
\hline 20 & 18,00 & 32,40 & 24,67 & 4,83 & 21,9 \\
\hline 30 & 25,90 & 44,40 & 35,37 & 6,41 & 20,4 \\
\hline 50 & 46,80 & 61,60 & 52,62 & 5,29 & 18,1 \\
\hline 100 & 99,30 & 99,90 & 99,80 & 0,22 & 14,5 \\
\hline
\end{tabular}

However, some efforts are still required to improve appropriate indicator combinations that show similar optical characteristics but different luminescence lifetimes depending on the analyte to be measured.

\section{ACKNOWLEDGMENT}

The authors would like to thank PreSens Precision Sensing $\mathrm{GmbH}$, Regensburg, Germany, for technical support, especially I. Klimant and C. Huber, for sensor and optical setup preparation. The authors would also like to thank B. Grunwald for help with the DSP setup and Prof. Dr. Ing. W. Anheier for support of the M.S. thesis at the University of Bremen, Bremen, Germany.

\section{REFERENCES}

[1] G. Weber, "Resolution of the fluorescent lifetimes in a heterogeneous system by phase and modulation measurements," J. Chem. Phys. Chem., vol. 85, pp. 949-953.

[2] Z. Xu, S. Liao, J. Izatt, and J. Alcala, "Real-time frequency domain temperature and oxygen sensor with a single optical fiber," IEEE Trans. Biomed. Eng., vol. 44, pp. 1114-1121, 1997.

[3] E. A. D. Austin and J. P. Dakin, "Opto-electronic systems for addressing Ru oxygen sensors: Their design optimization and calibration process," in Proc. SPIE, 2001.
[4] J. R. Lakowicz, Principles of Fluorescence Spectroscopy, 2nd ed. Norwell, MA: Kluwer, 1999.

[5] C. Stehning and G. Holst, "A DSP-based measuring system for temperature compensated fiberoptical oxygen sensors," Proc. SPIE, vol. 4578-31, pp. 259-270, 2001.

[6] C. Stehning, "Aufbau eines DSP-gestuetzen signalverarbeitungssystems für temperaturkompensierte faseroptische sauerstoffsensoren," M.S. thesis, Univ. Bremen, Bremen, Germany, 2002.

[7] W. H. Press, Numerical Recipes in C: The Art of Scientific Computing. Cambridge, MA: Cambridge Univ. Press, 1999.

[8] G. Liebsch, I. Klimant, and O. S. Wolfbeis, "Luminescence lifetime temperature sensing based on sol-gels and poly(acrylonitrile)s dyed with ruthenium metal-ligand complexes," Adv. Mater., vol. 11, 1999.

[9] I. Klimant, M. Kühl, R. N. Glud, and G. Holst, "Optical measurement of oxygen and temperature in microscale: Strategies and biological applications," Sens. Actuators B, vol. 38-39, pp. 29-37, 1997.

[10] J. R. Alcala, S.-C. Liao, and J. Zheng, "Real time frequency domain fiberoptic temperature sensor," IEEE Trans. Biomed. Eng., vol. 42, pp. 471-476, Mar. 1995.

[11] J. N. Demas and B. A. DeGraff, On the Design of Luminescence Based Temperature Sensors, 1992.

[12] G. Holst, M. Kühl, I. Klimant, G. Liebsch, and G. Kohls, "Characterization and application of temperature microoptodes for use in aquatic biology," in Advances in Fluorescence Sensing Technology III, San Jose, CA, 1997.

[13] G. Holst, I. Klimant, M. Kühl, and O. Kohls, "Optical microsensors and microprobes," in Chemical Sensors in Oceanography, Ocean Science and Technology, M. Varney, Ed. Amsterdam, The Netherlands: Gordon and Breach, 2000, vol. 1, pp. 143-188.

[14] I. Klimant, V. Meyer, and M. Kühl, "Fiber-optic oxygen microsensors, a new tool in aquatic biology," Limnol. Oceanography, vol. 40, pp. $1159-1165,1995$.

[15] D. P. Papkovsky, J. Olah, I. V. Troyanovsky, N. A. Sadovsky, V. D. Rumyantseva, A. F. Mironov, A. I. Yaropolov, and A. P. Savitsky, "Phosphorescent polymer films for optical oxygen sensors," Biosens. Bioelectron., vol. 7, pp. 199-206, 1991.

[16] I. Klimant and G. Holst, "Optical Temperature Sensors and Optrodes with Optical Temperature Compensation," U.S. Patent 6303 386/EC Patent 96944 654, 2001.

[17] G. Liebsch, I. Klimant, and O. S. Wolfbeis, "Temperature-compensated mapping of oxygen distribution via time-resolved luminescence lifetime imaging using transparent optical sensor foils," Analyst, to be published.

Christian Stehning was born in 1975. He received the Diploma in electronic engineering from the University of Bremen, Bremen, Germany, in 2002, with a final work on a DSP-based signal processing system for hybrid fiber optical sensors. He is currently pursuing the Ph.D. degree on advanced motion correction techniques for magnetic resonance angiography (MRA) at Philips Research Laboratories, Hamburg, Germany.

From 1998 to 1999, he was with the Insitute for Microsensors, Actuators, and Systems (IMSAS), Bremen, as a Research Assistant working on ultrasonic distance sensors for automotive applications. From 2000 to 2002, he was with the Max Planck Institute for Marine Microbiology, Bremen, as a Research Assistant working on measuring devices and data transmission systems for fiberoptical oxygen sensors.

Gerhard A. Holst was born in 1962. He received the Diploma in electronic engineering from the RWTH University, Aachen, Germany, in 1990, with a final work about reflectance pulse oximetry with electrooptical and hybrid fiber optical sensors, and the Ph.D. degree from the Max Planck Institute (MPI) for Molecular Physiology, Dortmund, Germany, in 1994, on a new optical chemical sensing principle, the oxygen flux optode and its phase modulation based measuring system.

From 1994 to 2001, he was with the Microsensor Research Group, MPI, Bremen, Germany, as a Postdoctoral Scientist, where he worked on the development of new fiber optical microsensors, microoptodes, new measuring systems, and imaging systems for the application of planar optodes and their time resolved measuring schemes for laboratory and field applications. Since 2001, he has been with PCO AG, Kelheim, Germany, where he is responsible for research in camera systems for advanced scientific applications. 